\title{
Geometric Determination of the Spheres which Are Tangent to Four Given Ones
}

\author{
E. Roanes-Macías ${ }^{1}$, E. Roanes-Lozano ${ }^{1}$ \\ Universidad Complutense de Madrid, Dept. Algebra, \\ Edificio "La Almudena", c/ Rector Royo Villanova s/n, 28040-Madrid, Spain \\ roanes@mat.ucm.es ; eroanes@fi.upm.es
}

\begin{abstract}
Apollonius' problem (find the tangent circles to three given ones) has attracted many mathematicians and has been solved using different methods along more than 22 centuries. Nowadays computers allow to mechanize the solving process and to treat its generalization to higher dimension using algebraic methods. Starting from the classical Vieta-Steiner solution for dimension 2, we have developed a method valid for dimension $n$, that, thanks to the use of an original coding, allows to choose in advance the relative position of the solution sphere w.r.t. the given ones (i.e., if each tangency is exterior or interior). Moreover, the possible degeneracy of some of the solution (hyper-)spheres in (hyper-) planes and the existence of configurations with an infinity number of solutions are considered.
\end{abstract}

\section{Introduction}

More than 22 centuries ago Apollonius of Perga proposed in his book Contacts (Tangencies) the problem of constructing the tangent circles to three given ones.

The problem has interested many great mathematicians from antiquity up to date. The first known solution was found by F. Vieta [12] in 1600. Later, other solutions were found by Descartes, Newton, Euler, Steiner, Poncelet, Gergonne, Mannheim, Fouché... In 1898 Lemoine compared the simplicity and exactness of the different solutions found till then [4]. A discussion about the number of solutions of the problem was described by Hadamard [3].

The interest of the problem is not only aesthetic; it has also had practical applications. In 1936, Nobel award winner Soddy [11] particularized the problem to the study of the relation between the radius of a molecule and the radii of its atoms, finding a curious relation between them. This result was extended to dimension $n$ by Pedoe [6] and Coxeter [2] in 1968.

Also with a chemical motivation in the background, the extension of the problem to 3D (substituting circles by spheres and ellipsoids) has been recently solved by performing an algebraic reduction that makes use of Dixon resultant and Groebner bases [5].

Similarly, Maple's Geometry package uses a pure algebraic method to solve the problem. But these approaches are neither constructive nor allow the user 
to choose in advance the relative position of the solution circle w.r.t. the given ones (i.e., if it is externally or internally tangent to each given one).

A constructive solution of the $3 \mathrm{D}$ problem is given in this article. It uses a method, derived from Vieta-Steiner's, that allows to choose in advance the relative position of the solution sphere w.r.t. the four given ones. Also following this line, a constructive solution to the $2 \mathrm{D}$ case was developed by the authors some years ago $[9,10]$

\section{Solving the Problem in a Way that Allows to Choose the Solution in Advance}

Algebraic methods solve the problem by stating a polynomial system whose unknowns are the center and radius of the solution sphere and which coefficients depend on the coordinates of the centers and the radii of the four given spheres. The system is solved using a method that can deal with non-linear polynomial systems, like those based on Groebner bases.

As said above, these methods do not allow to choose in advance the relative position of the solution sphere w.r.t. the four given ones (i.e., if it is externally or internally tangent to each given one).

To achieve this goal we have decided to apply a geometric method, before coding it properly, in order to directly obtain the chosen solution when using the implementation.

\subsection{D Extension of Vieta-Steiner's Method}

Among the many existing geometric resolution methods, we have chosen the one that we consider fits best, according to its computational adaptability. It combines F. Vieta's reduction to a simpler problem, with the application of the inversion-based method due to J. Steiner.

So, Vieta-Steiner's solution of Apollonius' problem will be extended to 3D. The description of the 2D case can be found in any classic Euclidean Geometry book $[1,8,7]$. We shall introduce afterwards those concepts related to inversion that will make this computational approach possible.

In an Euclidean real space, the inversion of pole $O$ and power $k$ is the involutive transformation where two points, $P$ and $P^{\prime}$, different from $O$, do correspond iff $O, P, P^{\prime}$ are collinear points such that $\overrightarrow{O P} \cdot \overrightarrow{O P^{\prime}}=k$.

In this inversion, the sphere such that $P$ and $O$ are endpoints of a diameter, is the inverse of the perpendicular plane to $O P$ through $P^{\prime}$ (where $P^{\prime}$ is the inverse of $P$ ).

In the same inversion, the inverse of a sphere that doesn't pass through the pole of inversion, $O$, and such that $P$ and $Q$ are endpoints of a diameter and are collinear with $O$, is the sphere such that points $P^{\prime}$ and $Q^{\prime}$ (inverse of $P$ and $Q$, respectively) are endpoints of a diameter.

Let us state the problem precisely. Given four spheres $S_{0}, S_{1}, S_{2}, S_{3}$ of different centers $C_{0}, C_{1}, C_{2}, C_{3}$ and radii $r_{0}, r_{1}, r_{2}, r_{3}$ (respectively), another sphere, 
$S$, tangent to the four given ones and which center and radius will be denoted $C$ and $r$ (respectively), is to be determined.

Let us suppose $r_{0}=\min \left\{r_{0}, r_{1}, r_{2}, r_{3}\right\}$. When subtracting or adding $r_{0}$ from the radii of the four spheres $S_{0}, S_{1}, S_{2}, S_{3}$, another four spheres are obtained: $S_{0}^{\prime}, S_{1}^{\prime}, S_{2}^{\prime}, S_{3}^{\prime}$, of centers $C_{0}, C_{1}, C_{2}, C_{3}$ and radii $r_{0}^{\prime}=r_{0}-r_{0}=0, r_{1}^{\prime}=r_{1} \pm$ $r_{0}, r_{2}^{\prime}=r_{2} \pm r_{0}, r_{3}^{\prime}=r_{3} \pm r_{0}$ (whether adding or subtracting $r_{0}$ will depend on the inclusions between $S$ and $S_{1}, S_{2}, S_{3}$, as will be detailed below). They will be tangent to sphere $S^{\prime}$, of center $C$ and radius $r^{\prime}=r \pm r_{0}$ (depending on whether $S$ includes $S_{0}$ or not). This way the problem is reduced to determine the sphere $S^{\prime}$ through $C_{0}$ tangent to $S_{1}^{\prime}, S_{2}^{\prime}, S_{3}^{\prime}$. We shall refer to this first part of the process as Vieta's reduction.

Applying now an inversion, $I$, of pole $C_{0}$, the spheres $S_{1}^{\prime \prime}=I\left(S_{1}^{\prime}\right), S_{2}^{\prime \prime}=I\left(S_{2}^{\prime}\right)$ and $S_{3}^{\prime \prime}=I\left(S_{3}^{\prime}\right)$ are obtained. If precisely the geometric power of $C_{0}$ w.r.t. $S_{1}^{\prime}$ is chosen as the power of $I$, then $S_{1}^{\prime \prime}=S_{1}^{\prime}$.

As $S^{\prime}$ passes through the pole of inversion $C_{0}$ and $I$ is an angle-preserving transformation, figure $S^{\prime \prime}=I\left(S^{\prime}\right)$ must be a plane, tangent to spheres $S_{1}^{\prime \prime}, S_{2}^{\prime \prime}$ and $S_{3}^{\prime \prime}$ (curiously, the same configuration of a plane and three spheres is used in the 3D proof of a 2D theorem due to Gaspard Monge).

Consequently, the image by $I$ of a plane, $S^{\prime \prime}$, tangent to $S_{1}^{\prime \prime}, S_{2}^{\prime \prime}$ and $S_{3}^{\prime \prime}$, is a sphere or a plane (depending on whether $C_{0} \notin S^{\prime \prime}$ or $C_{0} \in S^{\prime \prime}$ ), $S^{\prime}$, that passes through $C_{0}$ and is tangent to $S_{1}^{\prime}, S_{2}^{\prime}$ and $S_{3}^{\prime}$. Unapplying Vieta's reduction to $S^{\prime}$, a solution sphere or plane, tangent to $S_{0}, S_{1}, S_{2}, S_{3}$, is obtained.

Clearly, from each plane $S^{\prime \prime}$, tangent to $S_{1}^{\prime \prime}, S_{2}^{\prime \prime}$ and $S_{3}^{\prime \prime}$, a solution sphere or plane would be obtained. As, in the usual case, there are at most 8 of such planes, and $S$ can include $S_{1}$ or not, the maximum number of solutions is 16 ( $S_{0}, S_{1}, S_{2}, S_{3}$ are supposed to be exterior two by two).

There are special cases where an infinite number of solutions exist. This is the case, for instance, when there is a cylinder or a cone that is tangent to the four spheres simultaneously or when $C_{0}, C_{1}, C_{2}, C_{3}$ are vertices of a square and $r_{0}=r_{1}=r_{2}=r_{3}$.

Up to here we have described a straightforward extension to $3 \mathrm{D}$ of VietaSteiner's method for solving the problem in the Euclidean plane. From here onwards our original contribution to solve the proposed problem begins. The main difficulty of the process lies on the appropriate selection of the tangent plane $S^{\prime \prime}$, in order to obtain precisely the desired solution for $S$, among the 16 possible ones. This will be achieved through an adequate coding.

\subsection{Coding the Geometric Elements}

A point $P$ of coordinates $\left(p_{1}, p_{2}, p_{3}\right)$ will be codified as the list of its coordinates: $\left[p_{1}, p_{2}, p_{3}\right]$.

The plane through point $P$ and perpendicular to the unitary vector $v$, of coordinates $\left(v_{1}, v_{2}, v_{3}\right)$, will be codified by the list $[P, v]$, where $P$ and $v$ are the sublists $\left[p_{1}, p_{2}, p_{3}\right]$ and $\left[v_{1}, v_{2}, v_{3}\right]$, i.e. by the list of lists $\left[\left[p_{1}, p_{2}, p_{3}\right],\left[v_{1}, v_{2}, v_{3}\right]\right]$.

The spherical surface of center $P$ and radius $r$ will be codified by list $[P, r]$, where $P$ is the sublist $\left[p_{1}, p_{2}, p_{3}\right]$ and $r$ is a positive real number. Therefore, the 
four spheres given in the problem will be introduced as a list $\left[S_{0}, S_{1}, S_{2}, S_{3}\right]$, where each $S_{i}$ is the sublist $\left[C_{i}, r_{i}\right]$, of its center and radius.

In order to determine the relative position of each of the given spheres, $S_{i} ; i=$ $0,1,2,3$, w.r.t the solution sphere, $S$, i.e., if they are included or not in $S$, an inclusion code, $g_{i}$, valued in $\{-1,1\}$ will be used. We shall assign $g_{i}=-1$ or $g_{i}=1$, depending on whether $S_{i}$ is external or internal tangent to the solution sphere $S$ (respectively). The relative position of the spheres in list $\left[S_{0}, S_{1}, S_{2}, S_{3}\right]$ w.r.t. the sphere solution, $S$, will be determined by a list of codes $\left[g_{0}, g_{1}, g_{2}, g_{3}\right]$.

\subsection{Selecting the Tangent Plane According to the Chosen Solution}

Once the four spheres $S_{0}, S_{1}, S_{2}, S_{3}$ are given, a right selection of the tangent plane $S^{\prime \prime}$ must be performed, in order to obtain precisely the desired solution for $S$, among all possible ones.

Let us denote by $v$ a unitary vector, ortogonal to this plane (director vector) and by $T_{1}, T_{2}, T_{3}$ the intersection points of this plane with the spheres $S_{1}^{\prime \prime}, S_{2}^{\prime \prime}, S_{3}^{\prime \prime}$ (respectively).

As $T_{1}, T_{2}, T_{3}$ belong to the tangent plane $S^{\prime \prime}$, vectors $\vec{T}_{1} T_{i} ; i=2,3$ are perpendicular to vector $v$, and therefore their dot products by vector $v$ are zero, so the following equalities will have to be verified.

$$
v \cdot v=1, \vec{T}_{1} T_{i} \cdot v=0 ; i=2,3
$$

On the other hand, according to the properties of inversion, sphere $S^{\prime}$ includes $S_{i}^{\prime}=I\left(S_{i}^{\prime \prime}\right)$ iff $S_{i}^{\prime \prime}$ is in a different half-space than the pole of inversion $C_{0}$ w.r.t. plane $S^{\prime \prime}=I\left(S^{\prime}\right)$. Therefore, for each pair of spheres among $C_{1}^{\prime \prime}, C_{2}^{\prime \prime}, C_{3}^{\prime \prime}$, they must be in the same or in different half-spaces of border $S_{i}^{\prime \prime}$, depending on whether their inclusion codes have the same or different signs. Consequently, the centers, $C_{1}^{\prime \prime}, C_{2}^{\prime \prime}, C_{3}^{\prime \prime}$ of the respective spheres $S_{1}^{\prime \prime}, S_{2}^{\prime \prime}, S_{3}^{\prime \prime}$, will be in one or the other half-space of border the tangent plane $S^{\prime \prime}$, depending on the values of $g_{i} ; i=1,2,3$. So, if $r_{1}^{\prime \prime}, r_{2}^{\prime \prime}, r_{3}^{\prime \prime}$ are the radii of the spheres $S_{1}^{\prime \prime}, S_{2}^{\prime \prime}, S_{3}^{\prime \prime}$ (respectively), their tangent points, $T_{1}, T_{2}, T_{3}$, can be expressed, initially, as

$$
T_{i}=C_{i}^{\prime \prime}+g_{i} r_{i}^{\prime \prime} v ; i=1,2,3
$$

Substituting now in (1) the values of $T_{1}, T_{2}, T_{3}$ given by (2), a polynomial system of degree 2 whose unknowns are the coordinates of vector $v$ is obtained. It is straightforward that this system is equivalent to another one where all equations are linear except one of degree 2, yielding two possible solutions for vector $v$.

This is logical. Let us observe that, once the values of the $g_{i}$ have been fixed, there are still two possible planes $S^{\prime \prime}$, tangent to $C_{1}^{\prime \prime}, C_{2}^{\prime \prime}, C_{3}^{\prime \prime}$, that keep these three spheres at the corresponding half-space of border $S^{\prime \prime}$ (in accordance with those values of $g_{i}$ ). Therefore there are two possible directions for vector $v$.

One of this two posible vectors $v$ has to be chosen. This selection must be done according to whether sphere $S_{1}^{\prime}$ is included or not in $S^{\prime}$ (what depends on 
whether $g_{1}$ is 1 or -1$)$. Consequently, sphere $S_{1}^{\prime \prime}=I\left(S_{1}^{\prime}\right)$ and the pole of inversion $C_{0}$ must be in different or the same half-space w.r.t. to the tangent plane $S^{\prime \prime}$, depending on whether $g_{1}$ is 1 or -1 .

But $S_{1}^{\prime \prime}$ and $C_{0}$ are in the same or in different half-space w.r.t. the tangent plane $S^{\prime \prime}$, depending on whether the inner products $\overrightarrow{T_{1} C_{1}^{\prime \prime}} \cdot v$ and $\overrightarrow{T_{1} C_{0}} \cdot v$ are of different or of the same sign.

Therefore, among the two different vectors $v$, the one such that the inner products $\overrightarrow{T_{1} C_{1}^{\prime \prime}} \cdot v$ and $\overrightarrow{T_{1} C_{0}} \cdot v$ are of different or the same sign will be chosen, depending on whether $g_{1}$ is 1 or -1 .

\subsection{Extension to Dimension $n$}

The whole process described above for dimension 3 is also valid for any dimension $n \geq 2$.

\section{Algorithm}

Input: $\left[S_{0}, S_{1}, S_{2}, S_{3}\right],\left[g_{0}, g_{1}, g_{2}, g_{3}\right]$ (list of spheres and list of inclusion codes) Output: $S$ (solution sphere(s), solution plane(s) or Without solution string)

(1) $S_{i}^{\prime}:=\left[C_{i}, r_{i}^{\prime}\right], r_{i}^{\prime}=r_{i}-r_{0} ; i=1,2,3 \quad$ (Vieta's reduction)

(2) $S_{i}^{\prime \prime}:=I\left(S_{i}^{\prime}\right)=\left[C_{i}^{\prime \prime}, r_{i}^{\prime \prime}\right] ; i=1,2,3 \quad\left(I=\right.$ inversion of center $\left.C_{0}\right)$

(3) $T_{i}:=C_{i}^{\prime \prime}+g_{i} r_{i}^{\prime \prime} v ; i=1,2,3$, where $v:=\left[v_{1}, v_{2}, v_{3}\right]$

(4) $\left[v^{*}, v^{* *}\right]:=$ solutions of system $\left\{v \cdot v=1, \vec{T}_{1} T_{i} \cdot v=0 ; i=2,3\right\}$

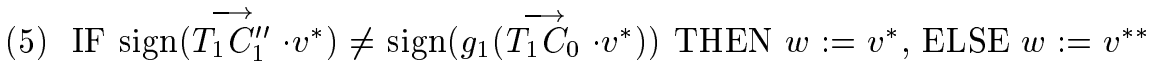

(6) IF $w$ imaginary THEN RETURN Without solution string

(7) $T_{1}:=C_{1}^{\prime \prime}+g_{1} r_{1}^{\prime \prime} w$ (tangent point of $S_{1}^{\prime \prime}$ )

(8) $S^{\prime \prime}:=\left[T_{1}, w\right]$ (tangent plane)

(9) IF $C_{0} \in S^{\prime \prime}$ THEN RETURN $S:=\left[C_{0}, w\right]$ (solution plane)

(10) $S^{\prime}:=I\left(S^{\prime \prime}\right)=\left[C, r^{\prime}\right]$ (inverse of the tangent plane)

(11) $S:=\left[C, r^{\prime}+g_{0} r_{0}\right] \quad$ (solution sphere; similarly solution spheres or plane(s))

\section{Implementation}

The previous algorithm does not require symbolic calculations but exact arithmetic (to be sure that the right decision is taken in the conditionals and for checking solutions). Anyway, as a system that contains a non-linear equation is to be solved, it is advisable to use a Computer Algebra System (CAS). Among 
them, we have chosen Maple, taking into account its comfort, diffusion, portability and calculation power.

The package we have developed works in any dimensión (for circles, spheres or hyper-spheres, respectively). It has 26 procedures, of which we shall briefly describe the main ones (those mentioned in the examples), omitting all subprocedures where the steps of the algorithm above are implemented.

From here onwards and for the sake of brevity, we shall talk of spheres really meaning circles or 3D-spheres or hyper-spheres, and we shall talk of planes really meaning lines or planes or hyperplanes (according to the dimension of the space).

ApoSol(SphereList,CodeList) is the main procedure. Its first argument is the list of spheres $\left[S_{0}, S_{1}, S_{2} \ldots\right]$ and its second argument is the list $\left[g_{0}, g_{1}, g_{2} \ldots\right]$ of inclusion codes of these spheres. It returns the solution sphere, $S$, as mentioned above:

- list of center and radius, $[C, r]$, if the solution is a sphere

- list of point and vector, $[P, v]$, if the solution is a plane

- string Without solution, if there is no real solution

- list of center and radius, expressed as a function of parameters (to which particular values should be given in order to obtain particular solutions), if there were infinite solutions.

ApoComp(Solution,SphereList,CodeList) is the procedure that allows to check if the solution obtained is correct and the right one. Its first argument is the solution, $S$, previously obtained, its second argument is the list $\left[S_{0}, S_{1}, S_{2} \ldots\right]$ of the given spheres and the third one is the list $\left[g_{0}, g_{1}, g_{2} \ldots\right]$ of inclusion codes. This procedure calculates the distances from the centers of the given spheres to the center of the solution sphere or to the solution plane, and subtracts from it the sum/difference of radii or distances, depending on the values of the $g_{i}$. If the solution is correct, such results must be zero all of them. So, a list of zeros must be obtained.

Equ(figure,vars) is a procedure that allows to obtain the equation of a figure of those considered in section 2.2 (if they are introduced in the way explained there). Using the terminology of 2.2 , its first argument is of the form $[P, r]$ (if the figure is a sphere) or of the form $[P, v]$ (if the figure is a plane). Its second argument is the list of names of the coordinate axes $[x, y, z \ldots]$ w.r.t. which the equation of $S$ is to be expressed (the output).

ApoDib(Solution,SphereList) allows to plot in Maple both the 2D and 3D cases. Its first argument is the solution (sphere or plane) and the second one is the list of given spheres. It returns the plot of the solution figure, $S$, together with all the given spheres.

\section{$5 \quad$ Gallery of Examples}

In the following examples the code is written in Maple 7. The solutions are obtained using ApoSol, and are allocated in variable Sol. They are checked af- 
terwards using procedure ApoComp. They are represented in Maple 7 using procedure $A$ poDib or using DPGraph2000 (a package specialized in graphing implicit 3D functions; see http://www.davidparker.com/index.html for details). When an equation is needed it is obtained using Equ.

Example 1. Given three circles, each one exterior to each other, determine the circle that is externally tangent to the first two ones and internally tangent to the third one.

$>\mathrm{S} 0:=[[2,-4], 3]: \mathrm{S} 1:=[[-5,3], 1]: \mathrm{S} 2:=[[2,5], 2]:$

$>\mathrm{S}:=[\mathrm{S} 0, \mathrm{~S} 1, \mathrm{~S} 2] ; \mathrm{G}:=[-1,-1,1]:$

$$
S:=[[[2,-4], 3],[[-5,3], 1],[[2,5], 2]]
$$

$>$ Sol: $=\operatorname{ApoSol}(\mathrm{S}, \mathrm{G})$;

$$
\text { Sol }:=\left[\left[\frac{-3126}{2455}+\frac{68}{2455} \sqrt{3619}, \frac{-103}{491}+\frac{28}{491} \sqrt{3619}\right], \frac{-4364}{2455}+\frac{252}{2455} \sqrt{3619}\right]
$$

$>\operatorname{ApoComp}(\mathrm{Sol}, \mathrm{S}, \mathrm{G})$;

$$
[0,0,0]
$$

$>\operatorname{ApoDib}(\mathrm{S}, \mathrm{Sol})$;

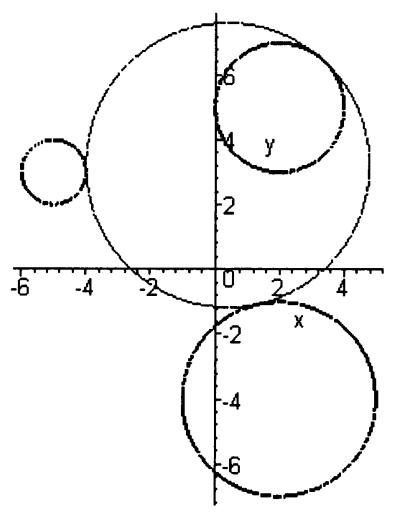

Fig. 1. Figure of Example 1

Example 2. Given four spheres, each one exterior to each other, determine the sphere externally tangent to them all.

$>\mathrm{S} 0:=[[0,5,0], 2]: \mathrm{S} 1:=[[0,-5,0], 3]: \mathrm{S} 2:=[[5,0,0], 2]:$

$>\mathrm{S} 3:=[[0,0,5], 1]: \mathrm{S}:=[\mathrm{S} 0, \mathrm{~S} 1, \mathrm{~S} 2, \mathrm{~S} 3] ; \mathrm{G}:=[-1,-1,-1,-1]:$

[[[0,5,0],2],[[0,-5,0],3],[[5,0,0],2], [[0,0,5],1]] 
$>$ Sol:=ApoSol $(\mathrm{S}, \mathrm{G})$;

Sol $:=\left[\left[\frac{1}{8048270}(4940+3 \sqrt{220667})(-279+2 \sqrt{220667}), \frac{1}{8048270}(4940+\right.\right.$ $\left.\left.3 \sqrt{220667})(-279+2 \sqrt{220667}), \frac{-98}{445}+\frac{3}{890} \sqrt{220667}\right], \frac{-457}{178}+\frac{1}{89} \sqrt{220667}\right]$

$>\operatorname{ApoComp}(\mathrm{Sol}, \mathrm{S}, \mathrm{G})$;

$$
[0,0,0,0]
$$

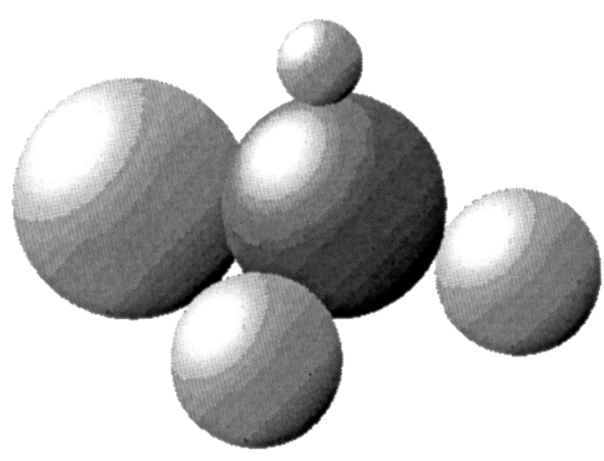

Fig. 2. Figure of Example 2

Example 3. Case where the solution sphere degenerates into a plane.

$>\mathrm{S} 0:=[[0,0,4], 3]: \mathrm{S} 1:=[[2,-7,-3], 4]: \mathrm{S} 2:=[[2,11,-3], 4]:$

$>\mathrm{S} 3:=[[-7,2,-3], 4]: \mathrm{S}:=[\mathrm{S} 0, \mathrm{~S} 1, \mathrm{~S} 2, \mathrm{~S} 3]: \mathrm{G}:=[-1,1,1,1]:$

$>\operatorname{Sol}:=\operatorname{ApoSol}(\mathrm{S}, \mathrm{G})$;

$$
\text { Sol:=[[2,- } 7,1],[0,0,-1]]
$$

$>\operatorname{ApoComp}(\mathrm{Sol}, \mathrm{S}, \mathrm{G})$;

$$
[0,0,0,0]
$$

$>\operatorname{Equ}(\mathrm{S} 0,[\mathrm{x}, \mathrm{y}, \mathrm{z}]), \operatorname{Equ}(\mathrm{S} 1,[\mathrm{x}, \mathrm{y}, \mathrm{z}]), \operatorname{Equ}(\mathrm{S} 2,[\mathrm{x}, \mathrm{y}, \mathrm{z}])$, $\mathrm{Equ}(\mathrm{S} 3,[\mathrm{x}, \mathrm{y}, \mathrm{z}])$;

$$
\begin{gathered}
x^{2}+y^{2}+z^{2}-8 z+7, x^{2}+y^{2}+z^{2}-4 x+14 y+6 z+46, \\
x^{2}+y^{2}+z^{2}-4 x-22 y+6 z+118, x^{2}+y^{2}+z^{2}+14 x-4 y+6 z+46
\end{gathered}
$$

$>\operatorname{Equ}(\operatorname{Sol},[\mathrm{x}, \mathrm{y}, \mathrm{z}])$;

$$
-z+1
$$




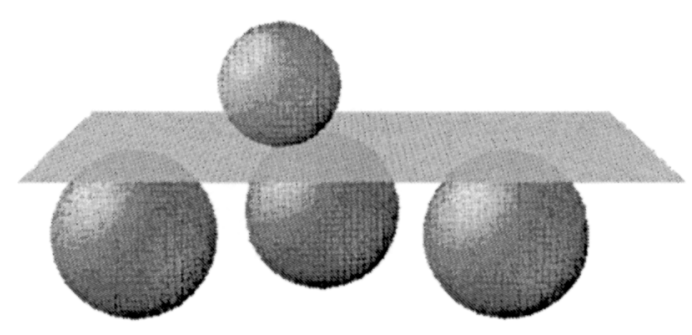

Fig. 3. Figure of Example 3

Example 4. Given four spheres, of which at least two are mutually external, try to determine a sphere internally tangent to them all.

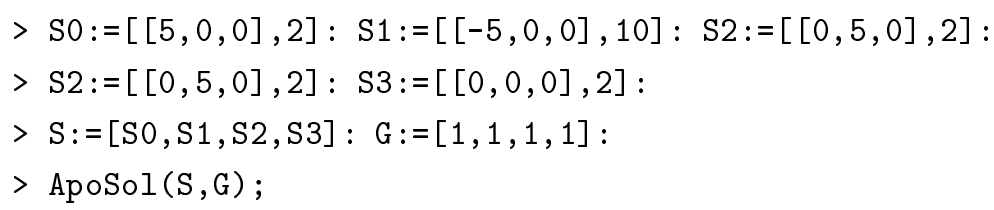

\section{Without solution}

Example 5. Given four non-intersecting spheres of the same radii and which centers are the vertices of a square, determine the spheres externally tangent to them all.

$>\mathrm{S} 0:=[[2,0,0], 1]: \mathrm{S} 1:=[[-2,0,0], 1]: \mathrm{S} 2:=[[0,2,0], 1]:$

$>\mathrm{S} 2:=[[0,2,0], 1]: \mathrm{S} 3:=[[0,-2,0], 1]:$

$>\mathrm{S}:=[\mathrm{S} 0, \mathrm{~S} 1, \mathrm{~S} 2, \mathrm{~S} 3]: \mathrm{G}:=[1,1,1,1]:$

$>\operatorname{Sol}:=\operatorname{ApoSol}(S, G)$;

$$
\text { Sol }:=\left[\left[0,0,2 \frac{t \sqrt{1-t^{2}}}{-1+t^{2}}\right], 2 \sqrt{-\frac{1}{-1+t^{2}}}-1\right]
$$

(They are spheres which center lies on line $x=0=y$ and which radii are $\geq 1$ ) $>\operatorname{ApoComp}(\mathrm{Sol}, \mathrm{S}, \mathrm{C})$;

$$
[0,0,0,0]
$$

Example 6. Given five hyper-spheres in 4D, determine a hyper-sphere tangent to them all.

$>\mathrm{S} 0:=[[0,5,0,0], 2]: \mathrm{S} 1:=[[0,0,0,0], 1]: \mathrm{S} 2:=[[0,0,5,0], 2]:$

$>\mathrm{S} 3:=[[-5,0,0,0], 1]: \mathrm{S} 4:=[[0,0,0,5], 1]:$

$>\mathrm{S}:=[\mathrm{S} 0, \mathrm{~S} 1, \mathrm{~S} 2, \mathrm{~S} 3, \mathrm{~S} 4]: \mathrm{G}:=[1,-1,1,-1,-1]:$ 
$>\operatorname{Sol}:=\operatorname{ApoSol}(\mathrm{S}, \mathrm{G})$;

$$
\begin{gathered}
\text { Sol }:=\left[\left[\frac{-5}{2}, \frac{1}{12334}(375+8 \sqrt{862})(96+5 \sqrt{862}), \frac{1}{12334}(375+8 \sqrt{862})\right.\right. \\
\left.(96+5 \sqrt{862}), 5 / 2], \frac{41}{7}+\frac{5}{14} \sqrt{862}\right]
\end{gathered}
$$

$\operatorname{ApoComp}(\operatorname{Sol}, \mathrm{S}, \mathrm{G})$;

$$
[0,0,0,0,0]
$$

\section{Conclusions}

Mixing new and old techniques has made possible to generalize Apollonius' problem to higher dimension. The approach maintains Vieta-Steiner's solution's elegance. The original coding of each solution (hyper-)sphere is another advantage of this approach. Both the resolution method and the implemented program are valid for dimension $n$ (hyper-spheres in dimension $n$ tangent to $n+1$ given ones). Moreover, the possible degeneracy of some of the solution (hyper-)spheres and the possibility of an infinity of solutions are considered. Therefore we think this is one more step forward in the study of Apollonius' problem.

\section{Acknowledgements}

This work is partially supported by project TIC2000-1368-C03-01 (Ministry of Science and Technology, Spain).

\section{References}

1. Berger M.: Geometry I, Springer-Verlag, Berlin-Heidelberg, 1987.

2. Coxeter H.S.M.: The Problem of Apollonius, Am. Math. Monthly, Vol. 75 (1968) $5-15$.

3. Hadamard J.: Lecons de Géométrie Elementaire, A. Colin, Paris, 1947-49.

4. Lemoine E.: Application de d'une méthode d'évaluation de la simplicité des constructions a la comparaison de quelques solutions du probléme d'Apollonius, Nouvelles Ann. Math. (1892) 453-474.

5. Lewis R. H.: Apollonius Meets Computer Algebra. In: Proceedings of ACA'2001, http://math.unm.edu/ACA/2001/Proceedings/NonStd/

6. Pedoe D.: On a theorem in Geometry, Am. Math. Monthly, Vol. 74 (1967) 627-640.

7. Pedoe D.: Geometry, Dover Pub., New York, 1988.

8. Ogilvy C.S.: Excursions in Geometry, Dover Pub., New York, 1990.

9. Roanes Lozano E.: El Problema de Apolonio, Bol. Soc. Puig Adam, Vol. 14 (1987) 13-41.

10. Roanes Macías E., Roanes Lozano E.: Nuevas tecnologías en Geometría, Editorial Complutense, Madrid, 1994.

11. Soddy F.: The Kiss Precise, Nature, 137 (1936) 1021.

12. Vieta F.: Varia Responsa. IX: Apollonius Gallus, Real Academia de Ciencias, Madrid, not dated edition (Reprint of the original dated 1600). 